\title{
List of publications of Lothar Geitler (since 1979)
}

(continuing the list published in Österr. Bot. Z. 116: 6-17, 1969, and P1. Syst. Evol. 131: $166-168,1979$.

345. Einige kritische Bemerkungen zu neuen zusammenfassenden Darstellungen der Morphologie und Systematik der Cyanophyceen. - Pl. Syst. Evol. 132: 153- 160 (1979).

346. Zur Lebensgeschichte der Diatomee Achnanthes linearis und Bemerkungen über andere Achnanthes-Arten. - Pl. Syst. Evol. 132: 231 - 238 (1979).

347. Zur Kenntnis der Saprolegniale Ectrogella eunotiae. - Sydowia 31: 38 - 43 ("1978" 1979).

348. Beiträge zur Entwicklungsgeschichte und Taxonomic einiger Achnanthes-Arten, Subgenus Microneis (Bacillariophyceae). - Pl. Syst. Evol. 134: 1-10 (1980).

349. Chroococcopsis epiphytica n. sp., eine neue Cyanophycee aus dem Gebiet des Neusiedler Sees. - Pl. Syst. Evol. 136: 53-62 (1980).

350. Die Conjugate Mesotaenium dodekahedron GeITrer von einem zweiten Fundort. Linzer biol. Beitr. 11: 295-296 (1980).

351. Zellteilung und Bildung von Innenschalen bei Hantzschia amphioxys und Achnanthes coarctata. - Pl. Syst. Evol. 136: 275-286 (1980).

352. Die Lage des Chromatophors in Beziehung zur Systematik von Cymbella-Arten ( $B a-$ cillariophyceae). - P1. Syst. Evol. 138: 153-156 (1981).

353. Die infraspezifischen Sippen von Cocconeis placentula des Lunzer Seebachs. - Arch. Hydrobiol. Suppl. 63 (Algological Studies 30): 1- 11 (1982).

354. Eine bemerkenswerte Oscillatoriacee, Katagnymene accurata n. sp. (Cyanophyceae). - Pl. Syst. Evol. 140: $293-306$ (1982).

355. Zur Kenntnis der Oscillatoriacee Pseudanabaena galeata (Cyanophyta). - Pl. Syst. Evol. 141: $169-175$ (1982).

356. Eine Anabaena-artige Cyanophycee mit auffallendem Zellteilungs- und Fadenzerfallsrhythmus. - Pl. Syst. Evol. 142: 129-135 (1983).

357. Ergänzungen zu älteren Listen der Typen der Auxosporenbildung pennater Diatomeen. - Arch. Hydrobiol. 101: $101-104$ (1984).

358. Automixis bei pennaten Diatomeen. - Pl. Syst. Evol. 150: 303-306 (1985).

359. Typification of Nitzschia flexoides (Bacillariophyceae). - P1. Syst. Evol. 156: 207-208 (1987). 